\title{
Assessing a Warping Methodology for the Identification of Increased Cardiovascular Risk Based on the HR Profile Morphology
}

\author{
Julia Ramírez ${ }^{1}$, Stefan van Duijvenboden ${ }^{2}$, Pablo Laguna ${ }^{3}$, Esther Pueyo ${ }^{3}$, Andrew Tinker ${ }^{1}$, Pier D \\ Lambiase $^{2}$, Patricia B Munroe ${ }^{1}$, Michele Orini ${ }^{4}$ \\ ${ }^{1}$ William Harvey Research Institute, Queen Mary University of London, London, United Kingdom \\ ${ }^{2}$ Institute of Cardiovascular Science, University College London, London, United Kingdom \\ ${ }^{3}$ BSICoS group, Aragon Institute of Engineering Research, University of Zaragoza, Zaragoza, Spain \\ ${ }^{4}$ Barts Heart Centre, St Bartholomews Hospital, London, United Kingdom
}

\begin{abstract}
Heart rate $(H R)$ response to exercise and recovery are strong predictors of cardiovascular mortality, but the HR profile morphology may add useful information for the identification of subjects at risk. Our aim was to characterise the HR profile morphology and assess its cardiovascular risk predictive value. 1-lead ECG recordings of 17,691 participants from the general population in an exercise stress test from the UK Biobank study were analyzed. A methodology based on time warping of the $H R$ profile was applied to compute the average HR profile morphology along the exercise test. Then, two series of warping-based morphological differences in amplitude, $d_{a}$, and time, $d_{w}$, were calculated by comparing each individual HR profile morphology with respect to this average $H R$ profile. Subjects who suffered one or more cardiovascular events showed significantly lower values of $d_{a}$ than survivors (median of $-10.5 \%$ vs $-7.5 \%, p=0.009$ ). Also, $d_{a}$ was significantly associated with cardiovascular mortality in a Cox model after adjusting for clinical variables, resting $H R$, difference between peak and resting $H R$, or between peak and recovery $H R$ after a follow-up period of five years $(p<0.0001)$. Individuals at risk show HR dynamics with slower adaptation to exercise than healthier subjects, possibly due to autonomic nervous system dysfunction.
\end{abstract}

\section{Introduction}

Several studies have demonstrated that indices of HR dynamics during exercise and recovery predict cardiac death $[1,2]$, and genetic studies are being undertaken on these phenotypes [3]. However, these measures only provide a rather simplistic description of the HR dynamics. We hypothesized that an index designed to better capture the HR profile morphology during the entire exercise stress test would show a better association with cardiovascular risk as compared to the HR responses to exercise or to recovery alone.

Two markers, $d_{w}$, and $d_{a}$, have been previously proposed to quantify single-lead T-wave morphological variability [4]. The amount of warping needed to remove the time domain variability, is measured by $d_{w}$, while $d_{a}$ quantifies the amplitude variability after removing the temporal domain variability. Both indices have been used to quantify T-wave morphological variations to predict sudden cardiac death $[5,6]$.

The aim of this study was (1) to quantify the morphological variability of the HR profile during an exercise stress test with respect to an average HR profile morphology using $d_{a}$ and $d_{w}$, (2) to assess their cardiovascular risk predictive value, and (3) to characterize the HR profile morphology across different risk groups based on the values of $d_{a}$.

\section{Materials and Methods}

\subsection{Materials}

This work has been performed using data from $\mathrm{N}=$ 17,691 participants in the Cardio physical fitness assessment within the UK Biobank (UKB) project from 2009 to 2013 [7]. The test uses cycle ergometry on a stationary bike in conjunction with a 1-lead ECG to record ECGs at rest (15 s pre-test), during graded activity (6 $\mathrm{min})$ and in recovery $(1 \mathrm{~min})$. The absolute maximum workload was calculated by adjusting for age, height, weight, resting HR and sex. The UKB study was approved by the North West Multi-Centre Research Ethics Committee and all participants provided written informed consent to participate in the UKB study.

Clinical data, including gender, age, body mass index (BMI), diabetic status, diastolic and systolic blood pres- 


\begin{tabular}{|c|c|c|c|c|}
\hline & $\begin{array}{l}\text { Overall population } \\
(n=17,691)\end{array}$ & $\begin{array}{c}\text { Survivors } \\
(n=17,356)\end{array}$ & $\begin{array}{l}\text { Cardiovascular risk } \\
\quad(n=335)\end{array}$ & $p$ value \\
\hline Gender [men] & $8,149(46.1 \%)$ & $7,914(45.6 \%)$ & $235(70.1 \%)$ & $<0.0001$ \\
\hline Age [years] & $59(13)$ & $59(13)$ & $63(8)$ & $<0.0001$ \\
\hline $\mathrm{BMI}\left[\mathrm{kg} / \mathrm{m}^{2}\right]$ & $26.5(5.58)$ & $26.37(5.33)$ & $27.96(5.68)$ & $<0.0001$ \\
\hline Diabetic & $873(5.0 \%)$ & $843(4.9 \%)$ & $30(9.0 \%)$ & 0.001 \\
\hline Smoker & $7,649(43.2 \%)$ & $7,484(43.1 \%)$ & $165(49.3 \%)$ & 0.026 \\
\hline DBP & $81.0(13.5)$ & $81(13)$ & $83.5(13)$ & 0.001 \\
\hline SBP & $136.0(24.5)$ & $136(24)$ & $141.5(24.8)$ & $<0.0001$ \\
\hline High cholesterol & $2,256(12.8 \%)$ & $2,186(12.6 \%)$ & $70(20.9 \%)$ & $<0.0001$ \\
\hline Maximum workload [Watt] & $80(40)$ & $80.0(30)$ & $90.0(39)$ & 0.015 \\
\hline Resting HR [bpm] & $71.0(15.33)$ & $71(15)$ & $71(16)$ & 0.939 \\
\hline$\Delta H R^{\mathrm{ex}}[\mathrm{bpm}]$ & $42.0(16.5)$ & $42(16)$ & $37(16)$ & $<0.0001$ \\
\hline$\Delta H R^{\mathrm{rec}}[\mathrm{bpm}]$ & $30.0(13.0)$ & $31(14)$ & $27(14)$ & $<0.0001$ \\
\hline$d_{a}[\%]$ & $-7.6(21.0)$ & $-7.5(21.0)$ & $-10.5(20.6)$ & 0.007 \\
\hline$d_{w}[\mathrm{~s}]$ & $-1.5(1.8)$ & $-1.5(1.8)$ & $-1.1(1.8)$ & 0.009 \\
\hline
\end{tabular}

Table 1. Clinical variables and HR profile indices in the general population

sure (DBP and SBP), and cholesterol levels were collected the day of the exercise protocol (Table 1).

The primary endpoint of this work was cardiovascular risk, including death or admissions to hospital due to coronary artery disease, heart failure, arrhythmias, cardiovascular disease or ischaemia, and conduction disorders. Data from health records and death certificates were sent to UKB on a quarterly basis up to March 2016.

\subsection{Methods}

\subsubsection{Derivation of the Heart Rate Profiles}

The HR measurements were available for downloading from UK Biobank. The HR profile represents the HR dynamics during the bike test and is defined as a function of time, $\boldsymbol{f}_{i}^{s}\left(\boldsymbol{t}_{i}^{s}\right)$, where $\boldsymbol{t}_{i}^{s}=\left[t_{i}^{s}(1), \ldots, t_{i}^{s}\left(N_{s}\right)\right]^{\top}$ and $N_{s}$ being the total duration of $\boldsymbol{t}_{i}^{s}$ in samples, obtained by filtering each $i$-th instantaneous $\operatorname{HR}(i=1, \cdots, \mathrm{N})$ with a median filter over 15 beats to eliminate respiratory sinus arrhythmia and low frequency oscillations [8], after interpolating and low pass filtering at $4 \mathrm{~Hz}$.

Each individual HR profile was normalised (subtracting the resting HR and dividing by the standard deviation) prior to further analysis to exclusively capture variations in the HR profile morphology, and make the morphological indices independent from the resting HR, peak exercise HR or HR at recovery.

\subsection{Mathematical Framework}

Let $\boldsymbol{f}^{r}\left(\boldsymbol{t}^{r}\right)=\left[f^{r}\left(t^{r}(1)\right), \ldots, f^{r}\left(t^{r}\left(N_{r}\right)\right)\right]^{\top}$ and $\boldsymbol{f}^{s}\left(\boldsymbol{t}^{s}\right)=\left[f^{s}\left(t^{s}(1)\right), \ldots, f^{s}\left(t^{s}\left(N_{s}\right)\right)\right]^{\top}$ be two HR profiles, where $\boldsymbol{t}^{r}=\left[t^{r}(1), \ldots, t^{r}\left(N_{r}\right)\right]^{\top}$ and $\boldsymbol{t}^{s}=$ $\left[t^{s}(1), \ldots, t^{s}\left(N_{s}\right)\right]^{\top}$ and $N_{r}$ and $N_{s}$ being the total duration of $\boldsymbol{t}^{r}$ and $\boldsymbol{t}^{s}$, respectively, in samples. We take $\boldsymbol{f}^{r}\left(\boldsymbol{t}^{r}\right)$ as the reference HR profile and $\boldsymbol{f}^{s}\left(\boldsymbol{t}^{s}\right)$ as the HR profile to be compared with respect to $\boldsymbol{f}^{r}\left(\boldsymbol{t}^{r}\right)$.

Let $\gamma\left(\boldsymbol{t}^{r}\right)$ be the warping function that relates $\boldsymbol{t}^{r}$ and $\boldsymbol{t}^{s}$, such that the composition $\left[\boldsymbol{f}^{s} \circ \gamma\right]\left(\boldsymbol{t}^{r}\right)=\boldsymbol{f}^{s}\left(\boldsymbol{\gamma}\left(\boldsymbol{t}^{r}\right)\right)$ denotes the time domain warping of $\boldsymbol{f}^{s}\left(\boldsymbol{t}^{s}\right)$ using $\gamma\left(\boldsymbol{t}^{r}\right)$.

The square-root slope function (SRSF), $\boldsymbol{q}_{f}(\boldsymbol{t})$, of a function $\boldsymbol{f}(\boldsymbol{t})$ is defined as the square-root of the derivative of $\boldsymbol{f}(\boldsymbol{t})$, considering the sign [9]. Then, as demonstrated in [10], the optimal warping function is the one that minimizes the amplitude difference between the SRSF of $\boldsymbol{f}^{r}\left(\boldsymbol{t}^{r}\right)$ and $\boldsymbol{f}^{s}\left(\boldsymbol{\gamma}\left(\boldsymbol{t}^{r}\right)\right), \boldsymbol{q}_{f^{r}}\left(\boldsymbol{t}^{r}\right)$ and $\boldsymbol{q}_{\left[f^{s} \circ \gamma\right]}\left(\boldsymbol{t}^{r}\right)=$ $\boldsymbol{q}_{f^{s}}\left(\boldsymbol{\gamma}\left(\boldsymbol{t}^{r}\right)\right) \sqrt{\dot{\dot{\gamma}\left(\boldsymbol{t}^{r}\right)}}$, respectively [9]:

$\boldsymbol{\gamma}^{*}\left(\boldsymbol{t}^{r}\right)=\underset{\boldsymbol{\gamma}\left(\boldsymbol{t}^{r}\right)}{\arg \min }\left(\left\|\boldsymbol{q}_{f^{r}}\left(\boldsymbol{t}^{r}\right)-\boldsymbol{q}_{f^{s}}\left(\boldsymbol{\gamma}\left(\boldsymbol{t}^{r}\right)\right) \sqrt{\dot{\gamma}\left(\boldsymbol{t}^{r}\right)}\right\|(\boldsymbol{)})\right.$

The dynamic programming algorithm was used to obtain the solution of this optimization [11].

We used a previously defined metric, $d_{w}$ [4], to quantify the level of warping needed to optimally align any two HR profiles:

$$
d_{w}=\frac{1}{N_{r}} \sum_{n=1}^{N_{r}}\left|\gamma^{*}\left(t^{r}(n)\right)-t^{r}(n)\right| .
$$

The normalised amplitude difference between $\boldsymbol{f}^{r}\left(\boldsymbol{t}^{r}\right)$ and $\boldsymbol{f}^{s}\left(\boldsymbol{\gamma}^{*}\left(\boldsymbol{t}^{r}\right)\right)$ is quantified as:

$$
d_{a}=\frac{\frac{1}{N_{r}} \sum_{n=1}^{N_{r}} f^{s}\left(\gamma^{*}\left(t^{r}(n)\right)\right)-f^{r}\left(t^{r}(n)\right)}{\frac{1}{N_{r}} \sum_{n=1}^{N_{r}} f^{r}\left(t^{r}(n)\right)} \times 100 .
$$

\subsubsection{Mean warped $H R$ profile}

From the set of $N$ HR profiles, we calculated an initial mean warped HR profile that is an optimal representative average both in temporal and amplitude domains. The 


\begin{tabular}{|c|c|c|c|c|}
\hline & \multicolumn{2}{|c|}{ Univariate } & \multicolumn{2}{|c|}{ Multivariate } \\
\hline & $\begin{array}{l}\text { Hazard ratio } \\
(95 \% \mathrm{CI})\end{array}$ & $p$ value & $\begin{array}{l}\text { Hazard ratio } \\
(95 \% \mathrm{CI})\end{array}$ & $p$ value \\
\hline Gender [men] & $2.75(2.18-3.47)$ & $<0.0001$ & $3.55(2.56-4.93)$ & $<0.0001$ \\
\hline Age [per 1 SD increment] & $1.76(1.55-2.00)$ & $<0.0001$ & $1.56(1.33-1.82)$ & $<0.0001$ \\
\hline BMI [per 1 SD increment] & $1.29(1.17-1.42)$ & $<0.0001$ & $\mathbf{1 . 3 3}(1.19-1.49)$ & $<0.0001$ \\
\hline Diabetic & $1.99(1.37-2.87)$ & $<0.0001$ & $1.49(1.01-2.20)$ & 0.049 \\
\hline Smoker & $1.29(1.04-1.59)$ & 0.022 & $1.00(0.80-1.26)$ & 0.969 \\
\hline DBP [per 1 SD increment] & $1.18(1.06-1.31)$ & 0.002 & $0.97(0.83-1.14)$ & 0.697 \\
\hline SBP [per 1 SD increment] & $1.38(1.24-1.53)$ & $<0.0001$ & $1.05(0.92-1.20)$ & 0.511 \\
\hline High cholesterol & $1.85(1.42-2.40)$ & $<0.0001$ & $1.19(0.90-1.57)$ & 0.225 \\
\hline Maximum workload [per 1 SD increment] & $1.14(1.03-1.27)$ & 0.016 & $\mathbf{0 . 7 2}(0.60-0.87)$ & 0.001 \\
\hline$\Delta H R^{\mathrm{ex}}[$ per $1 \mathrm{SD}$ decrement] & $1.39(1.23-1.56)$ & $<0.0001$ & $1.08(0.94-1.22)$ & 0.261 \\
\hline$\Delta H R^{\text {rec }}[$ per $1 \mathrm{SD}$ decrement] & $1.30(1.16-1.47)$ & $<0.0001$ & $0.91(0.78-1.08)$ & 0.253 \\
\hline$d_{a}$ [per 1 SD decrement] & $1.16(1.04-1.29)$ & 0.009 & $1.31(1.16-1.47)$ & $<0.0001$ \\
\hline
\end{tabular}

Table 2. Association of clinical variables and HR profile indices with cardiovascular risk

methodology is explained in detail in [4]. We, then, recalculated the mean warped HR profile by only considering those individual HR profiles highly correlated (Spearman's correlation coefficient $>0.98$ ) with the initial mean warped HR profile.

\subsubsection{HR profiles morphological differences}

The morphological differences between each i-th individual HR profile and the mean warped HR profile were quantified using $d_{a}$ and $d_{w}$ [4], creating $\boldsymbol{d}_{\boldsymbol{a}}=$ $\left[d_{a}[1], \cdots, d_{a}[N]\right]$ and $\boldsymbol{d}_{\boldsymbol{w}}=\left[d_{w}[1], \cdots, d_{w}[N]\right]$.

We additionally calculated the HR response to exercise $\left(\Delta H R^{\mathrm{ex}}\right)$ as the difference between the peak HR and the resting $H R$, and the $H R$ response to recovery $\left(\Delta H R^{\mathrm{rec}}\right)$ as the difference between the peak HR and the recovery HR. Resting HR was defined as the mean HR during pre-test period, peak HR as the maximum HR during exercise, and recovery $\mathrm{HR}$ as the minimum HR 1 minute after the peak exercise [3].

\subsubsection{Risk HR Profile Morphologies}

The population was divided into five balanced groups according to the five quintiles of $d_{a}$ and, in each group, a HR profile representative of the average HR dynamics for that group along the exercise test was computed using the same warping method described in [4].

\subsection{Statistical Analyses}

Two-tailed Mann-Whitney and Fisher exact tests were used for univariate comparison of quantitative and categorical data, respectively, to evaluate the association of the clinical variables and indices derived from the HR profiles with the primary endpoint.

Univariate and multivariate Cox regression analyses were performed to determine the predictive value of the clinical variables and indices derived from the HR profiles. Backward analysis was applied with a retention criterion of $P<0.05$. A value of $P<0.05$ was considered statistically significant. Statistical analyses was performed using SPSS version 25.0 (SPSS Inc, Chicago, IL).

\section{Results and Discussion}

Figure 1 shows the individual normalized HR profiles after time warping with the same mean warped HR profile displayed in red bold. As it can be seen, the time warping method corrects for the difference in duration of each individual protocol. Thanks to the warping algorithm, the HR profiles from these individuals can be still included in the analyses, thus avoiding reduction in the sample size.

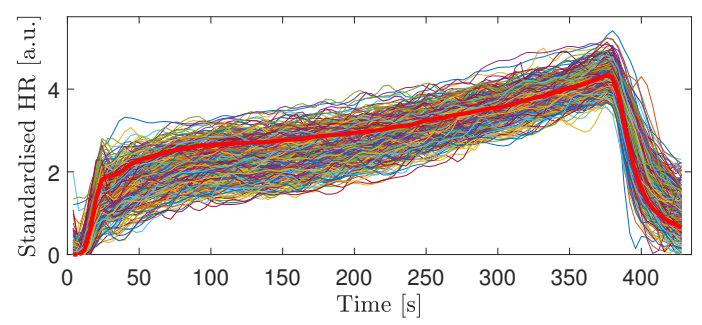

Figure 1. $\boldsymbol{f}^{r}\left(\boldsymbol{t}^{r}\right)$ (bold red) superimposed to $\boldsymbol{f}^{s}\left(\boldsymbol{\gamma}\left(\boldsymbol{t}^{r}\right)\right)$.

During the follow-up period there were 335 (1.9\%) deaths or hospitalisations due to cardiovascular disease. Upon comparison of clinical variables between survivors and cardiovascular risk groups (Table 1), there were significantly more men, diabetics, smokers, and subjects with high cholesterol in the cardiovascular risk group as compared to survivors. This group was also characterised by a significantly higher age, BMI, DBP and SBP. Analysis of ECG markers during exercise shows that individuals in this group had a significantly higher predicted maximum workload and $d_{w}$ values, but significantly lower $\Delta H R^{\text {ex }}$, 
$\Delta H R^{\text {rec }}$, and $d_{a}$ values. Resting HR was not significantly different between both groups.

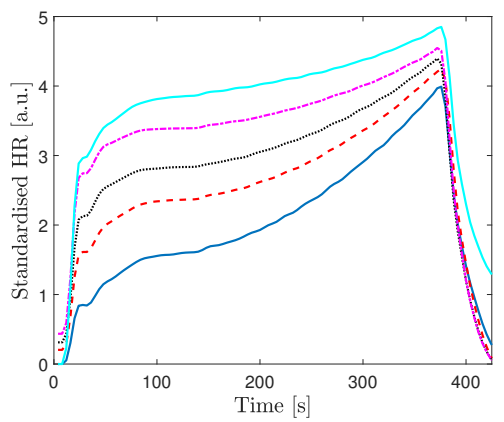

Figure 2. Five most representative HR profile morphologies corresponding to the first (blue), second (dashed red), third (dotted black, corresponding to the mean warped HR profile), fourth (dashed-dotted magenta) and fifth (cyan) quintiles of $d_{a}$.

Univariate Cox analysis revealed that decrements in $\Delta H R^{\mathrm{ex}}, \Delta H R^{\mathrm{rec}}$ and $d_{a}$ were associated with cardiovascular risk, contrary to resting HR of $d_{w}$ (Table 2). Multivariate Cox proportional hazard models were constructed by adjusting for all variables that were significantly associated with cardiovascular risk in the univariate model (Table 2). The index $d_{a}$ remained significantly associated with increased cardiovascular risk, independently of gender, age, BMI, diabetic status and the maximum workload. The indices $\Delta H R^{\mathrm{ex}}$ and $\Delta H R^{\mathrm{rec}}$ lost their significant association.

The five mean warped HR profiles, representative of the HR dynamics within each risk group defined by the $d_{a}$ quintiles are shown in Figure 2. Individuals in the first quintile of $d_{a}$ (blue HR profile) had a hazard ratio of 1.48 $(95 \%$ CI 1.52-210, $\mathrm{p}=0.012)$ compared to those in the fifth quintile (cyan HR profile) in the Multivariate Cox model.

\section{Conclusions}

The morphology of the HR profile during an exercise test provides information independent of the resting, peak and recovery HR for cardiovascular event and mortality risk prediction. Individuals at risk show HR dynamics with slower adaptation to exercise than healthier subjects possibly due to an attenuated effect of the sympathetic nervous system on HR or a reduced chronotropic competence. Further studies will explore the contribution of genetics on the morphology of HR profiles.

\section{Acknowledgements}

This research has been conducted using the UK Biobank Resource (application 8256) and is supported by the MRC grant MR/N025083/1. JR acknowledges support from the People Programme (Marie Curie Actions) of the European Union's Seventh Framework Programme (FP7/2007/2013) under REA grant agreement 608765. EP acknowledges support of projects DPI2016-75458-R (MINECO) and ERC-2014-StG 638284 (ERC). MO is supported by an IEF 2013 Marie Curie fellowship. We also wish to acknowledge the NIHR Cardiovascular Biomedical Research Unit at Barts and QMUL, UK.

\section{References}

[1] X. Jouven et. al., "Heart-rate profile during exercise as a predictor of sudden death," N Engl J Med, vol. 352, no. 19, pp. 1951-1958, 2005.

[2] A.S. Adabag et. al., "Relation of heart rate parameters during exercise test to sudden death and all-cause mortality in asymptomatic men," Am J Cardiol, vol. 101, pp. 14371443, 2008.

[3] Ramírez J., van Duijvenboden S., Ntalla I., et. al., "Thirty loci identified for heart rate response to exercise and recovery implicate autonomic nervous system," Nat Comms, p. In Press, 2018.

[4] Ramírez J. et al., "Variability of ventricular repolarization dispersion quantified by time-warping the morphology of the T-waves.," IEEE Trans Biomed Eng, vol. 64, no. 7, pp. 1619-1630, 2017.

[5] Ramírez J. et. al., "T-wave morphology restitution predicts sudden cardiac death in patients with chronic heart failure," J Am Heart Assoc, vol. 6, no. 5, p. e005310, 2017.

[6] Ramírez J. et. al., "Sudden cardiac death and pump failure death prediction in chronic heart failure by combining ECG and clinical markers in an integrated risk model," PLoS ONE, vol. 12, no. 10, p. e0186152, 2017.

[7] C. Bycroft et. al., "Genome-wide genetic data on 500,000 UK Biobank participants," bioRxiv.

[8] Orini M. et. al., "Long-term intra-individual reproducibility of heart rate dynamics during exercise and recovery in the UK Biobank cohort,” Plos One, vol. 12, p. e0183732, 2017.

[9] J. D. Tucker et. al., "Generative models for functional data using phase and amplitude separation," Comput Stat Data Anal, vol. 61, pp. 50-66, 2013.

[10] Ramírez J. et al., "An index for T-wave pointwise amplitude variability quantification.," Computing in Cardiology Conference (CinC), pp. 49-52, 2016.

[11] D. P. Bertsekas, Dynamic programming and optimal control, ch. 1. Belmont, MA, 1995.

Address for correspondence:

Julia Ramírez, Charterhouse Square,

London EC1M 6BQ, United Kingdom

j.ramirez@qmul.ac.uk 